\title{
The mechanism analyzing and use of corner Reflector against anti-ship
}

\section{missiles}

\author{
WANG Li ying ${ }^{1, a}$, JIANG Ning ${ }^{2}$ and SUN Yi ${ }^{3, b}$ \\ ${ }^{1}$ Dept.of Postgraduate Management, Dalian Naval Academy, Dalian116018, China \\ ${ }^{2}$ Dept.of Information Warfare Studies, Dalian Naval Academy, Dalian 116018, China \\ ${ }^{3}$ Unit 92896 of Navy, Dalian116018 \\ a547743958@qq.com, $\underline{\text { b38261135@qq.com }}$
}

Keywords: corner reflector; target search; operational use.

\begin{abstract}
As one of the passive jamming methods, the corner reflector has wide frequency band and strong RCS stability. Through analyzing the anti-ship missiles searching process, anti-ship missiles object recognitions, mechanism of corner reflector, we analyze the use of three jamming method, confusion jamming, dilution jamming, and centroid jamming in naval battlefield.
\end{abstract}

\section{Introduction}

Anti-ship missiles are the major weapons in modern naval battlefield, and they make the great threat to the navy surface ships[1]. With the technology of the anti-ship missiles guidance develop rapidly, it makes great challenge to the electronic interference. The searching method is the key of the anti-ship missiles guidance technology. It not only affects the ratio of target-catch, it also affects the ways of missiles firing, attack and tactics used.

\section{Anti-ship missiles target search}

\subsection{Missiles searching principle}

Nowadays, anti-ship missiles develop fast, the ways of targets hunting of anti-ship missiles have changed from "hunting the one which is searched" to multiple ways, such as "targets recognition"[2].

After missiles fly to the automatic control terminal, the terminal guidance radar will open and search the targets. This is the missiles searching process. The missiles searching process include range searching and azimuth searching. These two kinds of searching methods work together. The missiles range searching is through the terminal guidance radar searching gate move periodically to hunt targets echo. The starting position of the searching gate is random. If the searching gate hunts the targets, the targets are hunted by range searching method. The missiles azimuth searching is using radar beam to scan in space. After opening the terminal guidance radar, the antenna beam will search from right side of the missiles axis to the left side of the missiles axis in the space. When the antenna beams reach the limit of left side, it will work in reverse. Then, circulates the work. If the radar beam covered the targets, it means that the targets have been caught in space. When the targets meet the hunting conditions in both range searching and azimuth searching, targets echo intensity and the number of targets echo also meet the conditions, the radar will be sure of hunting the targets. 
Now, most missiles have taken track while scan system. When the radar finds the targets, it will track the targets and continue search the airspace at the same time. If the missiles find the targets in the searching, it will make sure the target is existed and transmit the information of orientation, elevation and distance to the computers. If it is the true targets, radar will move to the tracking condition and track wile scan condition.

\subsection{Influence factors of missiles target recognition}

Now, missile terminal radar has the ability to recognize the targets. The missile target recognition, is mainly refers to the missile terminal guidance radar to give instruction for missile precision selected target which is according to the different characteristics of radar target to test, enhance, filter, analytic processing the target scattering or reflecting which is detected, to rule out all kinds of interference information, and extract target information which will be attacked. The longer the missile range is, the greater the maybe position and searching area is.

Now, the major factors which affect the ability of the anti-ship missile target selection are:

1. Missile searching and catching parameter

Different missiles, the specific searching and catching parameters will directly affect the size of missile extended figure, actual searching and catching area. Whether can catch the target or not and the number of the hunting targets are the precondition of targets choosing.

2. Missile target selection mechanism

Now, among the missile target selection mechanism, the relative position selection and the echo intensity selection are two common selections. Both two selections mechanism have using restriction. For example, when we use the echo intensity selection mechanism, if the decoys echo intensity of enemy greater than the intended targets, the selection fails; If we use the relative position selection mechanism, such as the "left side target" principle, the target's preset position when the missile opened is requested. But for the intermediate-range anti-ship missiles, because of the long self-controlled phase, the possibility of the target maneuvers is higher, it will be more difficulty for people to accurately predict the relative position of the missile and the target.

3. The relative position of missile and target

The relative position of missile and target can also influence the ability of the missile selection. Mainly includes precision of target designation, target maneuver and so on. They will also affect the selection result.

4. Radar echo characteristics of target and the interference ability

False target is one of the main jamming methods of affecting the selecting ability of the missile; it includes active jamming and passive jamming. Passive jamming uses the similar characteristic of actual target to confuse anti-ship missiles guidance system and cover vessels; Active jamming is using the outboard active suppressing jamming or clutter interference and many other methods to decoy missile and protect our vessels.

Now, the fundamental of anti-ship missile terminal guidance radar recognition in the world is using the target's RCS information of amplitude, phase, frequency-shift, waveform and polarization to extract target characteristics or image information to select the target.

\section{Working principle of the corner reflector}

\subsection{The basic working principle of the corner reflector}

Corner reflector is usually composed of several metal planes. It is a radar false target and could 
give strong reflection of incident radar echo in a very wide incident angle range. From the electronic warfare, corner reflector is passive countermeasure equipment[3]. Corner reflector is easy to produce and equip, it can interfere different systems radar and all horizontal directions radar. It is an effective measure of anti-air.

Corner reflector is composed of three metal plates which are intersecting vertically. In a very wide incident angle range, the incident electromagnetic wave will reflect three times, then reflected in original direction. Its maximum reflection direction is the center axis of the corner reflector. The angle of center axis and three vertical axis is equal and all about 54.75 degrees. In this direction, corner reflector has the maximum RCS. The principle of triangular corner reflector is shown in the figure 1.

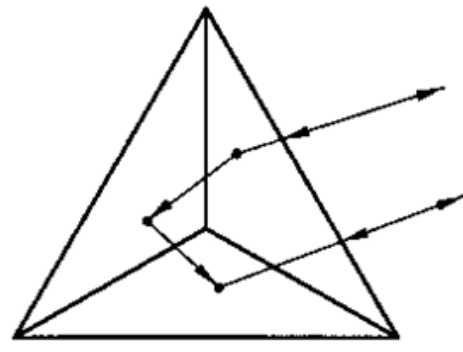

Figure.1 principle of triangular corner reflector

The maximum RCS available from corner reflector is proportional to four power of the edge, and inversely proportional to the square of the wavelength. According to the composition of the corner reflector plate's shape, it can be divided into triangular corner reflector, circular corner reflector and quadrate corner reflector. Among these three kinds of corner reflectors, if the edges are same, the triangular corner reflector has the minimum RCS, the circular corner reflector is the second, and the quadrate corner reflector has the maximum RCS.

$$
\begin{aligned}
& \sigma_{\Delta \max }=4.19 \frac{\alpha^{4}}{\lambda^{2}} . \\
& \sigma_{\text {Omax }}=15.6 \frac{\alpha^{4}}{\lambda^{2}} . \\
& \sigma_{\square \max }=37.3 \frac{\alpha^{4}}{\lambda^{2}} .
\end{aligned}
$$

The formula (1), (2), (3) are the maximum RCS available from triangular, circular and quadrate corner reflector. $\alpha$ is a variable, $\lambda$ is the incident wavelength. The Figure 1 is the relationship between area and edge length of triangular, circular and quadrate corner reflector.

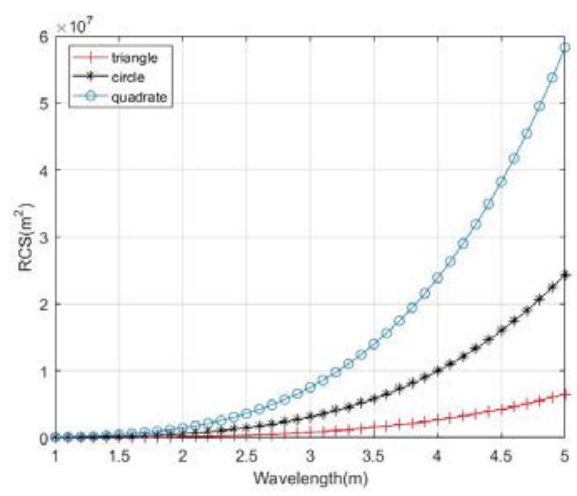

Fig. 1 Relationship between Area and Edge Length Of Three Kinds Of Corner Reflectors. Form the figure1, the quadrate corner reflector's RCS is the biggest when three kinds of corner 
reflector's edges are the same. Compared with other passive jamming equipment, corner reflector has wide frequency band and strong RCS stability. It has the advantages of staying in air for a long time, having little influence with sea battlefield and having the ability to confront various radars.

\section{The operational use of corner reflector}

Integration of hard and soft weapons anti-missile is the basic way of navy surface ships operation in international. Passive jamming as one of the electronic countermeasures measures, plays an important role in air and missile defense. As one of the passive jamming method, although the corner reflector haven't equipped in China, comparing with other passive jamming equipment, corner reflector has wide frequency band, strong RCS stability. And at the same time, it can stay in air for a long time, have little influence with sea battlefield and have the ability to confront various radars. It has the great advantages in the operational use[4].

\subsection{Confusion jamming}

Under the modern naval battle condition, in order to cover themselves better, both sides use beyond-the- horizon attack. So it is very important to make battlefield camouflage in both defense and attack. The beyond-the-horizon anti-ship missile uses air early warning aircraft or reconnaissance aircraft to guide; it brings great threat to our vessels. Compared with other ship-based passive jamming method, corner reflector has the high similarity echo characteristic and long action time. Also, it can work as confusion jamming to interfere enemy early-warning system especially to enemy air-platform[5].

In the fight against the enemy early-warning system, we can use corner reflection to disguise encampment and sea battlefield. It has the ability to confuse enemy early-warning aircraft, attacking aircraft and air-platform. Compared with chaff, corner reflector has long action time, similarity echo and better jamming effect.

\subsection{Dilution jamming}

Dilution jamming is usually used to counter the missile terminal guided system which is in the searching stage. It is a kind of operational use which is needed to put the decoy in a long distance. Through deceiving the anti-ship missile terminal guided radar, the decoys let the missile intercept and follow them in order to protect the ships. Ship-bone jamming system needs the information of missile, the ship itself and meteorological environment to ensure the jamming plan. Then put the corner reflector in special position and direction, in order to form several decoys around the ship. Due to the large RCS and long action time, corner reflector can be put quickly as soon as we receive the enemy attacking information. It can save time for ship moving. As shown in the figure 2.

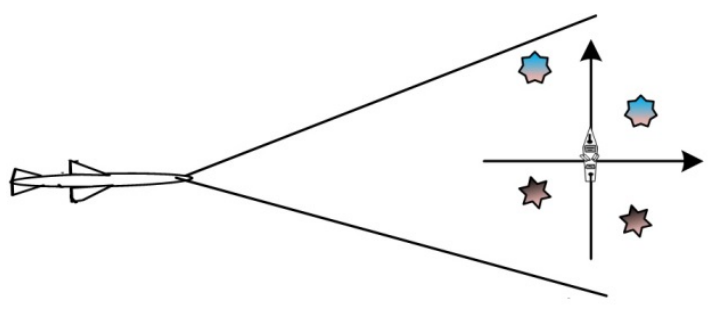

Fig. 2 The Diagram Of The Corner Reflector Dilution Jamming 


\subsection{Centroid jamming}

Centroid jamming is a passive jamming that is using to against the terminal guided radar which is in the tracking section. The terminal guided radar opens to capture the tracked ships. After the radar warning equipment measures the terminal guided radar signal, it will transfer the signal to the passive jamming equipment. According to the missile incoming parameters, wind direction, wind speed and the course of our ship, the control equipment will launch the corner reflector to the same resolution cell of terminal guided radar tracking system. In this way, the terminal guided radar will follow the center energy between the corner reflector and the ship instead of ship itself. With the moving of the ship, the terminal guided radar's tracking point will falls on the corner reflector and far from the ship. In this way, it will protect the ship. Because of the great available RCS from corner reflector and the little influence from the wind direction and wind seep, the jamming effect is much better. $\mathrm{O}$ is the centroid point in Figure 3.

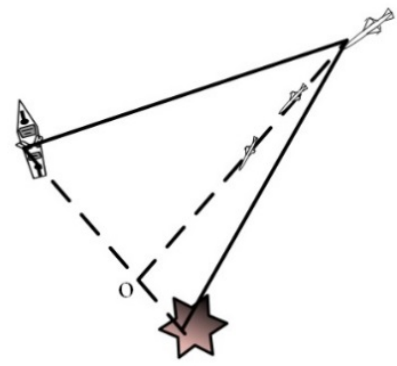

Fig. 3 The Diagram of The Corner Reflector Dilution Centroid Jamming

\section{The conclusion}

Corner reflector as one of the important passive jamming method, has the characteristic of long action time, similarity radar echo of the ship and many others. It has great jamming effect in the air and missile defense. This essay analyses the missile searching process and three jamming types of corner reflector. It has the reference value to the battle.

\section{Acknowledgements}

This work is supported by China Postdoctoral Science Foundation under Grant No.2016M602962 and No. 2017 T100797.

\section{References:}

[1] Guoqing Zhao. Principle of Radar Countermeasures[M]. Xi'an Electronic University Publications, Xi’an, 2003.

[2] Guanghui Wang, Xuguang Xin, Liping Zhang. Simulation Model of Anti-Ship Missile Terminal Guided Radar Searching Process[J]. Technology of Tactical Missile, 2014(5):49-53.

[3] Gdesser T, Balanls C A. Backscatter Analysis of Dihedral Corner Reflector Using Physical Optic and The Physical Theory of Diffraction[J]. IEEE Trans of Antennas Propag, 35(10), 1987.

[4] Gang Chai, Yimin Lu, Xiaoming Chen: Tactical Operational of Corner Reflector In Navy[J]. Shipboard Electronic Countermeasure, 29(5), 2006.

[5] Jing Chen. Passive Radar Jamming Principle[M]. National Defense Industry Press, Beijing, 2009. 